\title{
Influence of dietary conjugated linoleic acid isomers and selenium on growth, feed efficiency, and liver fatty acid profile in rats
}

\author{
K. Korniluk ${ }^{1}$, M. Czauderna ${ }^{1,3}$, J. Kowalczyk ${ }^{1}$, A. Mieczkowska ${ }^{1}$, \\ M. Taciak ${ }^{1}$ and L. Leng ${ }^{2}$ \\ ${ }^{I}$ The Kielanowski Institute of Animal Physiology and Nutrition, Polish Academy of Sciences \\ 05-110 Jabłonna, Poland \\ ${ }^{2}$ Institute of Animal Physiology, Slovak Academy of Sciences \\ 04001 Košice, Slovak Republic
}

(Received 10 October 2005; accepted 6 January 2006)

\begin{abstract}
The influence of dietary CLA isomer(s) and/or selenized yeast on the growth, concentration of CLA isomers and other fatty acids in the liver was investigated in rats. Plasma blood triacylglycerols (TAG), total cholesterol (TC), HDL and LDL cholesterol fractions in relation to dietary CLA isomer(s) and/or selenium (Se) were analysed. The experiment was performed on female rats (Wistar), 8 weeks of age and initial body weight of about $200 \mathrm{~g}$. After a 1-week preliminary period, for 4 weeks the animals were fed a diet enriched in conjugated linoleic acid (CLA) isomer(s) and selenized yeast $(2 \times 2$ experimental design). Dietary Se or/and CLA isomer(s) resulted in small changes in the spleen, heart, kidneys and brain, and increased liver weight. Administration of Se and trans10cis12CLA most efficiently increased the body weight gain of rats. CLA isomer(s) administered with or without Se elevated the CLA isomer(s) level in the liver. These results demonstrate that trans,transCLA isomers are metabolized more slowly, while cis, trans/trans, cisCLA isomers, more rapidly to longchain fatty acids containing a conjugated double bond. Enrichment of the diet in CLA isomer(s) with or without Se caused a reduction in the capacity of $\Delta 9-, \Delta 6$ - and $\Delta 5$-desaturases in the liver, while dietary trans 10cis 12CLA or the CLA isomer mixture increased $\Delta 4$-desaturases. The contents of oleic acid, C20:4n-6, and C20:5n-3 decreased in the liver, whereas the level of C22:5n-3 and C22:6n-3 increased in the liver of rats fed the CLA isomer mixture. Individual dietary CLA isomers with or without Se increased the concentration of palmitic and stearic acids in the liver. All experimental diets increased the concentration of triacylglycerols in blood plasma, while trans10cis12CLA with or without Se usually decreased the concentration of total cholesterol, LDL, and HDL cholesterol.
\end{abstract}

KEY WORDS: conjugated linoleic acid isomers, selenium, cholesterol, liver, blood plasma, fatty acids, rats

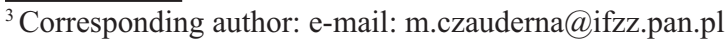




\section{INTRODUCTION}

Selenium (Se) is a trace bio-element essential for living organisms, occurring at about $10 \mathrm{mg} \mathrm{Se} / 60 \mathrm{~kg}$ human body weight (Suzuki, 2005). A greater percentage of seleno-methionine (Se-Met) is incorporated non-specifically into animal proteins in place of methionine (Met), particularly efficiently in a low Met diet (Tapiero et al., 2003), as tRNAMet does not discriminate between Met and Se-Met. Stored Se-Met can be a reserve pool of Se and much evidence indicates that Se deficiency stimulates the metabolism of Se-Met to other chemical forms of Se like seleno-cysteine (SeCys) (Whanger, 2004; Suzuki, 2005). Half of Se-proteins have been implicated in antioxidant functions, and Se-Cys is essential in the active centres of Se-enzymes that carry out redox reactions, e.g., glutathione peroxidase (GPx), thyroid hormone deiodinase families, or thioredoxin reductase (Tapiero et al., 2003; Suzuki, 2005). GPx acts synergistically with tocopherol and provides a second line of defence against hydroperoxides, which can damage membranes and other cell structures (Tapiero et al., 2003). Recently it has been reported that an adequate Se level in animal and human diets protects against lipid peroxidation and accumulation of carbonyl moieties on protein produced by oxidative stress (Tapiero et al., 2003). Feeding diets with selenized yeast (Se-yeast; 60\% Se as Se-Met (Rayman, 2004)) to animals results in effective induction of GPx activity, indicating that the animal converts this chemical form of Se to Se-Cys that is essential for the synthesis of Se-proteins (Whanger, 2004). Considering the above evidence, we decided to investigate whether a diet enriched in Se-yeast stimulated the accumulation of conjugated linoleic acid (CLA) isomers in rat livers. CLA isomers are believed to play a principal role in many important physiological functions and may lead to changes in body composition, body fat reduction, overall energy expenditure or modulate immune function (Banni et al., 2004; Rainer and Heiss, 2004). CLA isomers are also expected to inhibit tumorigenesis by interfering in the metabolism of some carcinogens (Wahle et al., 2004). Therefore, the objective of our studies was to determine the effects of Se-yeast on the CLA isomer and other fatty acid concentrations in the liver of rats. Furthermore, the influence of the experimental diet enriched in Se and/or CLA isomer(s) on the body gain of rats, organ weight and feed intake was also determined.

\section{MATERIAL AND METHODS}

\section{Animals and treatments}

Ten groups of female rats (Wistar, Ifz: BOA), 8 weeks of age with an initial body weight of about $200 \mathrm{~g}$, were housed individually in plastic cages as described by Czauderna et al. $(2004 a, b)$. During a one-week preliminary period the animals 
were fed a standard Labofeed diet (Se content less than $0.1 \mathrm{ppm}$ ) given at a submaintenance level (daily $\sim 9 \mathrm{~g}$ of the diet per rat) to reduce their body fat (Table 1). In the experimental period lasting 4 weeks, rats were fed ad libitum with the Labofeed diet enriched with a mixture of CLA isomers, the cis-9,trans-11CLA (c9t11), or trans-10,cis-12CLA (t10c12) isomer and/or Se, as Se-yeast (Rayman, 2004); the Se-yeast was donated by Sel-Plex, Alltech Inc. (USA). At the end of the experiment the rats were killed, blood samples collected, femoral muscles and livers removed, weighed, and analysed for fatty acid and/or mineral element content.

\section{Chemicals}

All reagents were analytical grade, whereas organic solvents were HPLC grade. The CLA isomer mixture, the cis-9, trans-11CLA and trans-10, cis-12CLA isomers were supplied by Larodan Fine Chemicals $\mathrm{AB}$ (Sweden). The purity and composition details of the CLA isomer mixture were examined using our $\mathrm{Ag}^{+}$-HPLC method (Czauderna et al., 2003a). n-Hexane (95\%) was purchased from Lab-Scan (Ireland), while all fatty acid standards and other chemicals were provided by SIGMA (USA).

\section{Saponification and fatty acid extraction}

The liver sample was frozen, lyophilized and the obtained residue was stored at $-20^{\circ} \mathrm{C}$ until analysis. The finely powdered liver sample $(\sim 50 \mathrm{mg})$ was placed in a vial and treated with a mixture of $2 \mathrm{ml}$ of $2 \mathrm{M} \mathrm{KOH}$ in water, $2 \mathrm{ml} 1 \mathrm{M} \mathrm{KOH}$ in methanol and $50 \mu \mathrm{l}$ of BHT solution in methanol $(20 \mathrm{mg} / \mathrm{ml})$. The mixture in a closed vial was then vigorously vortexed and heated under argon at $95^{\circ} \mathrm{C}$ for $10 \mathrm{~min}$, next cooled for $10 \mathrm{~min}$ at room temperature, and then sonicated for $10 \mathrm{~min}$. The obtained solution was protected from the light and stored in a sealed vial at $22-25^{\circ} \mathrm{C}$ overnight. Then, $3 \mathrm{ml}$ of water were added to the hydrolysate in the vial and the solution was again vigorously vortexed. The obtained solution was acidified with $4 \mathrm{M} \mathrm{HCl}$ to $\mathrm{pH} 1-2$ and free fatty acids (FAs) were extracted four times with $3 \mathrm{ml}$ of dichloromethane (DCM). The lower DCM layer was dried with $\sim 100 \mathrm{mg}$ of $\mathrm{Na}_{2} \mathrm{SO}_{4}$. To avoid any loss of free FAs, extraction was repeated 4 times using $3 \mathrm{ml}$ of n-hexane. Afterward, the upper n-hexane layer was combined with the DCM layer and the organic solvents were removed under a stream of argon. The concentration of assayed fatty acids was calculated from lyophilized liver samples.

\section{Preparation of fatty acid methyl esters (FA-MEs)}

To the residue, $2 \mathrm{ml}$ of $2 \mathrm{M} \mathrm{NaOH}$ in methanol and $50 \mu \mathrm{l}$ of BHT solution in methanol $(20 \mathrm{mg} / \mathrm{ml})$ were added while mixing, then flushed with argon, and 
reacted for $1 \mathrm{~h}$ at $80^{\circ} \mathrm{C}$. After cooling, $2 \mathrm{ml}$ of $25 \% \mathrm{BF}_{3}$ in methanol were added to the reaction mixture, flushed with argon, and heated for $1 \mathrm{~h}$ at $80^{\circ} \mathrm{C}$. To the cooled reaction mixture $5 \mathrm{ml}$ of water were added and then FA-MEs were extracted with $5 \mathrm{ml}$ of $\mathrm{n}$-hexane. The supernatant was transferred to a vial. Separation of FA-MEs was carried out by gas liquid-chromatography (GLC), while FA-MEs containing conjugated double bonds were also analysed using silver-liquid chromatography $\left(\mathrm{Ag}^{+}\right.$-HPLC) with photodiode array detection at $234 \mathrm{~nm}$ (Czauderna et al., 2003a).

\section{Analytical conditions}

The analyses of FA-MEs were performed on an Agilent 6890N GC equipped with a CP7489 fused silica capillary column $(100 \mathrm{~m} \times 0.25 \mathrm{~mm}$ i.d. $\times 0.2 \mu \mathrm{m}$ film thickness; Varian, USA) and FID, while split injection was performed using an Agilent 7683 autosampler; the $\mathrm{Ag}^{+}$-HPLC equipment was as previously described (Czauderna et al., 2003a). The FA-MEs were determined using GLC and $\mathrm{Ag}^{+}-$ HPLC according to Czauderna et al. (2005).

\section{Analysis of triacylglycerols, total, HDL and LDL cholesterol, and elements}

The concentrations of triacylglycerols (TAG) in blood plasma were quantified using Ektachem DT-60 kits donated by Johnson and Johnson Clinical Diagnostics Inc (i.e. microfiltration of deposited samples on Vitros-Slides and then a series of specific reactions with active reagents on the slides to produce coloured compounds quantified at $555 \mathrm{~nm}$, the intensity of the colour of these final products is proportional to the amount of TAG in assayed samples). Total cholesterol (TC), HDL and LDL cholesterol fractions in blood plasma were also determined using commercial Vitros-Slides test methodology (Johnson and Johnson Clinical Diagnostics Inc). Ten $\mu$ d drops of a sample are deposited on the Vitros-Slide. The sample spreads evenly and diffuses into the reagent layers. TC or HDL cholesterol fractions in the assayed plasma samples undergo a series of reactions in a VitrosSlide to produce coloured compounds. The intensity of these coloured products in analysed samples is proportional to the amount of TC (the detection at $555 \mathrm{~nm}$ ) and HDL cholesterol (detection at $660 \mathrm{~nm}$ ). The concentration of LDL cholesterol was calculated using the Friedwald equation (LDL cholesterol $=\mathrm{TC}-\mathrm{HDL}$ cholesterol - VLDL cholesterol).

The concentration of $\mathrm{Zn}, \mathrm{Cu}, \mathrm{Fe}, \mathrm{Mg}$ and $\mathrm{Ca}$ in lyophilized liver and femoral muscle samples was determined by flame (air-acetylene) atomic absorption spectrometry (PU9100X Atomic Absorption Spectrometer, UNICAM, Philips). 


\section{Statistical analyses}

The data are presented as means. Statistical analyses of the effects of Se or the CLA isomers in the diets were conducted using the nonparametric Mann-Whitney $\mathrm{U}$ test for comparing independent experimental groups, while statistical analyses of the interaction between the CLA isomer and Se were performed using twofactorial ANOVA analysis (the CLA isomer $\mathrm{x}$ Se; the experimental design $2 \times 2$ ). The Statistica ver. 6 and Excel 2003 programs were used in the statistical analyses. Differences were considered significant at the $\mathrm{P}<0.05$ or $\mathrm{P}<0.01$ level.

\section{RESULTS AND DISCUSSION}

The relationships between the experimental diets and feed intake, organ and body weight gain of rats are summarized in Table 1. In the current study, similarly as in our previous investigations (Czauderna et al., 2004b), no lesions or symptoms of Se-yeast and CLA isomer intoxication were found in rats fed experimental diets. In addition, after only 4 weeks of feeding experimental diets there were no considerable differences in the weight gain of rats and their organs among the groups. This indicates that any substantial differences in lipid and protein levels were not due to experimental diet intake and that the level of dietary organic Se was not toxic. The diet enriched in $1.2 \mathrm{ppm}$ of Se would not be toxic for rats as only chronic feeding of Se compounds at a rate of more than $5 \mathrm{ppm}$ can be hepatotoxic and teratogenic in animals and humans (Tapiero et al., 2003; Czauderna et al., 2004b). As can be seen from Table 1, feeding only t10c12CLA (Group $t 10 c 12)$ and the higher CLA isomer mixture dose (2\%) with or without Se to rats numerically or significantly reduced feed intake. These results are supported by several other studies, which have indicated that the higher CLA isomer mixture dose as well as $t 10 c 12 \mathrm{CLA}$ reduces feed intake, body weight gain of rats and increases energy expenditure (Czauderna et al., 2003b; Park et al., 2005). These results are supported by several other studies, which have indicated that due to its geometric and positional structure, the $t 10 c 12$ isomer in particular is the most potent CLA isomer in terms of antiobesity activity. Indeed, the hormones noradrenaline and adrenaline were significantly higher in experimental animals gavaged with the CLA isomer mixture, and data suggest that CLA isomers, especially t10c12CLA, enhance sympathetic nervous activity that leads to increased energy metabolism and eventual reduction of adipose tissue weight (Belury, 2002). Czauderna et al. (2003b) and Degrace et at. (2003) also reported that feeding rats or mice a diet supplemented with CLA isomers, particularly with the $t 10 \mathrm{c} 12$ isomer, increases fatty acid $\beta$-oxidation in the fat 


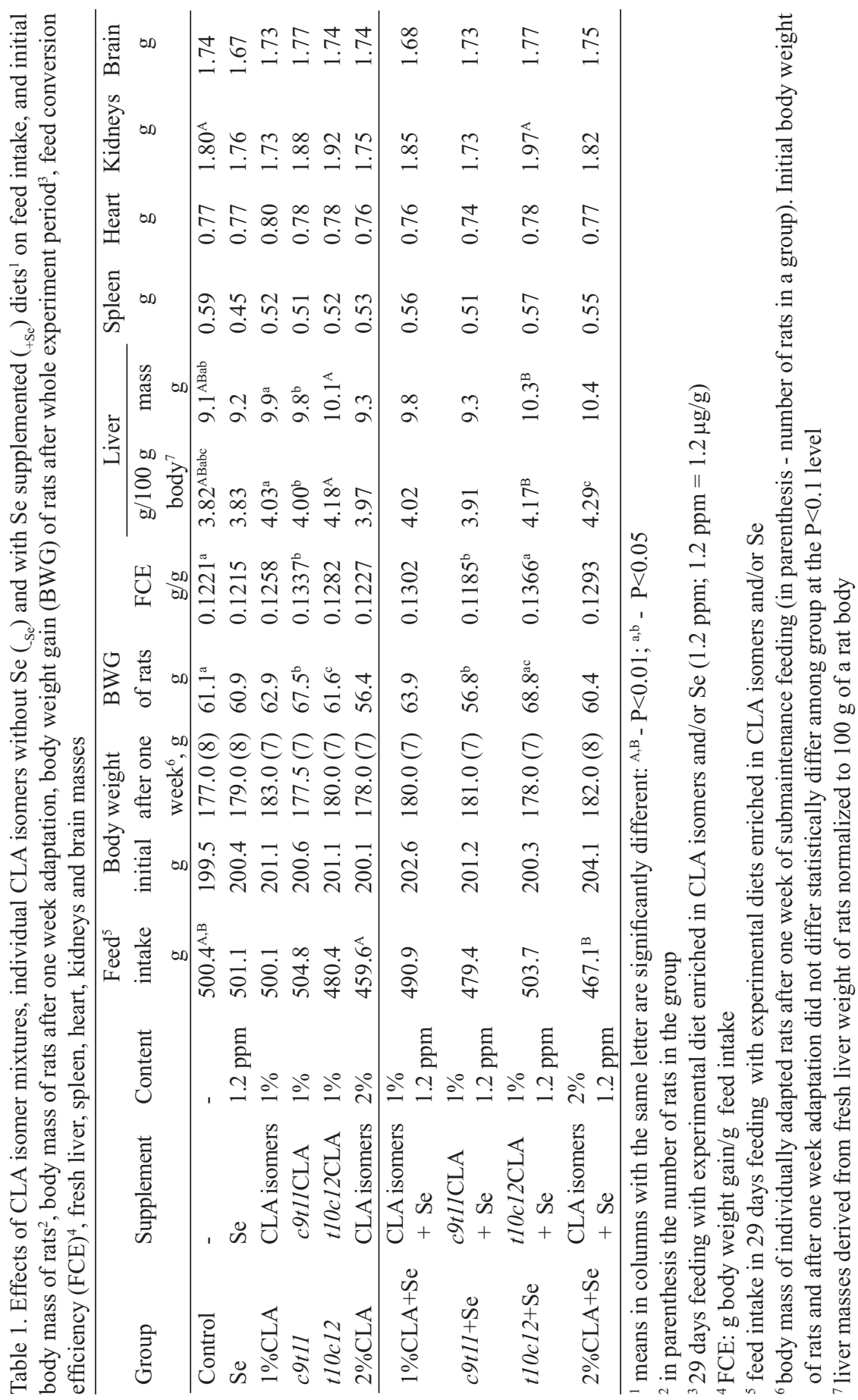


pad and in skeletal muscles (Alasnier et al., 2002), but not in the liver. The physiological responses to the CLA isomers in diets for rats suggest modulation of eicosanoid metabolism followed by elongation and $\Delta 5$-desaturation in the rat liver (Gnädig et al., 2001). Our data are consistent with these findings, thus feeding the CLA isomer mixture or the $t 10 c 12$ isomer, particularly simultaneously with Se, increased liver weight probably due to accumulation of fatty acid metabolites in this organ (Table 1). Moreover, a recent study (Simon et al., 2000) has shown that the content of protein in the liver of experimental animals fed CLA isomers increased. Another study also indicates that feeding $t 10 c 12 \mathrm{CLA}$ resulted in dramatic enlargement of the liver (Degrace et al., 2003). Indeed, at the peroxisomal level, considerable proliferation of small peroxisomes takes place together with increased capacity for $\beta$-oxidation of long-chain fatty acids (Terpsta et al., 2003). At the mitochondrial level, a greater part of activated fatty acids was directed from triacylglycerol esterification towards oxidation.

Interestingly, $t 10 c 12 \mathrm{CLA}$ is much more effective at lowering feed intake than the c9t11CLA isomer (Akahoshi et al., 2003), t10c12CLA appeared to be the effective isomer for modulating gene expression in, e.g., cultured 3T3-L1 pre-adipocytes (Choi et al., 2001). Consequently, no decrease in feed intake was observed when the diet was enriched only in the $c 9 t 11$ isomer, while administration of this isomer numerically increased body weight gain and feed conversion efficiency (FCE) compared with control rats. 110 c12CLA in the diet enriched in Se most efficiently increased the body weight gain (BWG) of rats, liver and kidneys weight and FCE, whereas the feed intake was similar to that by the control rats. Thus, our current results are in agreement with our previous studies on the interaction between $110 c 12 \mathrm{CLA}$ and inorganic Se (selenate), which also stimulated BWG, FCE, and liver weight in experimental rats (Czauderna et al., 2003b, 2004b).

Investigations into the effect of experimental diets on rat organ weight gain (Table 1) have demonstrated that CLA isomers with or without Se have no consistent influence on the weight of the spleen, heart, brain or kidneys (with the exception of the diet enriched in $t 10 c 12 \mathrm{CLA}$ and Se) and the concentration of $\mathrm{Zn}, \mathrm{Fe}$ and $\mathrm{Ca}$ in femoral muscle. Similarly to these results, all experimental diets resulted in inconsistent and negligible changes of the concentration of $\mathrm{Zn}, \mathrm{Cu}, \mathrm{Fe}$ and $\mathrm{Mg}$ in the liver (Table 2). Interestingly, the CLA isomers numerically elevated the concentration of $\mathrm{Ca}$ in the liver and the concentration of Fe in muscles; this effect was stronger as the concentration of CLA isomers in the diet increased. Our results are consistent with previous studies showing that CLA isomers mediated bone formation (Belury, 2002). In addition, the stimulating effect of CLA isomers on the accumulation of $\mathrm{Ca}$ in the liver is stronger if the diet is enriched in Se. 
Table 2. The concentration of $\mathrm{Zn}, \mathrm{Cu}, \mathrm{Fe}, \mathrm{Mg}$ and $\mathrm{Ca}$ in liver and muscles ( $\mu \mathrm{g} / \mathrm{g}$ of $\left.\mathrm{DM}^{1}\right)$ of rats fed CLA isomers and $\mathrm{Se}$

\begin{tabular}{|c|c|c|c|c|c|c|}
\hline Group & $\begin{array}{l}\text { Type of } \\
\text { Sample }\end{array}$ & $\mathrm{Zn}$ & $\mathrm{Cu}^{2}$ & $\mathrm{Fe}$ & $\mathrm{Mg}$ & $\mathrm{Ca}$ \\
\hline \multirow{3}{*}{ Control } & Liver & 102 & 5.90 & 660 & 594 & 78 \\
\hline & Muscle & 49 & - & 79 & 1616 & 199 \\
\hline & Liver & 96 & 5.93 & 705 & 589 & 81 \\
\hline \multirow[t]{2}{*}{$\mathrm{Se}$} & Muscle & 50 & - & 59 & 1606 & 187 \\
\hline & Liver & 94 & 5.24 & 618 & 581 & 81 \\
\hline \multirow[t]{2}{*}{$1 \% \mathrm{CLA}$} & Muscle & 52 & - & 89 & 1638 & 213 \\
\hline & Liver & 96 & 5.40 & 613 & 584 & 82 \\
\hline \multirow[t]{2}{*}{ c9t11 } & Muscle & 53 & - & 63 & 1672 & 234 \\
\hline & Liver & 100 & 5.17 & 659 & 584 & 88 \\
\hline \multirow[t]{2}{*}{$\mathrm{t} 10 \mathrm{c} 12$} & Muscle & 49 & - & 66 & 1630 & 198 \\
\hline & Liver & 93 & 4.94 & 700 & 596 & 87 \\
\hline \multirow[t]{2}{*}{$2 \%$ CLA } & Muscle & 48 & - & 107 & 1636 & 198 \\
\hline & Liver & 109 & 4.75 & 665 & 594 & 90 \\
\hline \multirow[t]{2}{*}{$1 \% \mathrm{CLA}+\mathrm{Se}$} & Muscle & 53 & - & 67 & 1544 & 255 \\
\hline & Liver & 119 & 7.06 & 699 & 618 & 92 \\
\hline \multirow[t]{2}{*}{$\mathrm{c} 9 \mathrm{t} 11+\mathrm{Se}$} & Muscle & 52 & - & 68 & 1648 & 287 \\
\hline & Liver & 98 & 5.00 & 661 & 603 & 88 \\
\hline \multirow[t]{2}{*}{$\mathrm{t} 10 \mathrm{c} 12+\mathrm{Se}$} & Muscle & 47 & - & 77 & 1624 & 197 \\
\hline & Liver & 105 & 7.41 & 688 & 603 & 97 \\
\hline $2 \% \mathrm{CLA}+\mathrm{Se}$ & Muscle & 50 & - & 106 & 1622 & 202 \\
\hline
\end{tabular}

${ }^{1}$ dry mass of liver and muscles

${ }^{2}$ the level of $\mathrm{Cu}$ in femoral muscles is below the quantification limit

The effect of the diets on the concentration of CLA isomers and other fatty acids in the liver

The current study also investigated the relationship between the experimental diets and the concentration of CLA isomers and other fatty acids in the liver (Table 4). As expected, diets containing CLA isomer(s), irrespective of the presence of extra Se, resulted in considerable accumulation of CLA isomer(s) in the liver and affected the concentration of fatty acids in rat liver compared with the control rats. Our current results (Tables 3 and 4) are in agreement with the observations of Alasnier et al. (2002) and our previous studies (Czauderna at al., 2003b, $2004 \mathrm{~b}$ ) in which $t 10 c 12 \mathrm{CLA}$ and $t 10 t 12 \mathrm{CLA}$ are more efficiently driven through $\beta$-oxidation in cells of the liver, muscles, kidneys or adipose tissue than their 9,11 homologues. Therefore, the value of ratio $\left(\mathrm{R}_{c 9 t / 1 \mathrm{CLA} / t \mid 0 c 12 \mathrm{CLA}}\right)$ of these isomers in the administered CLA isomer mixture $(\mathrm{R}=0.981)$ was lower compared with the 


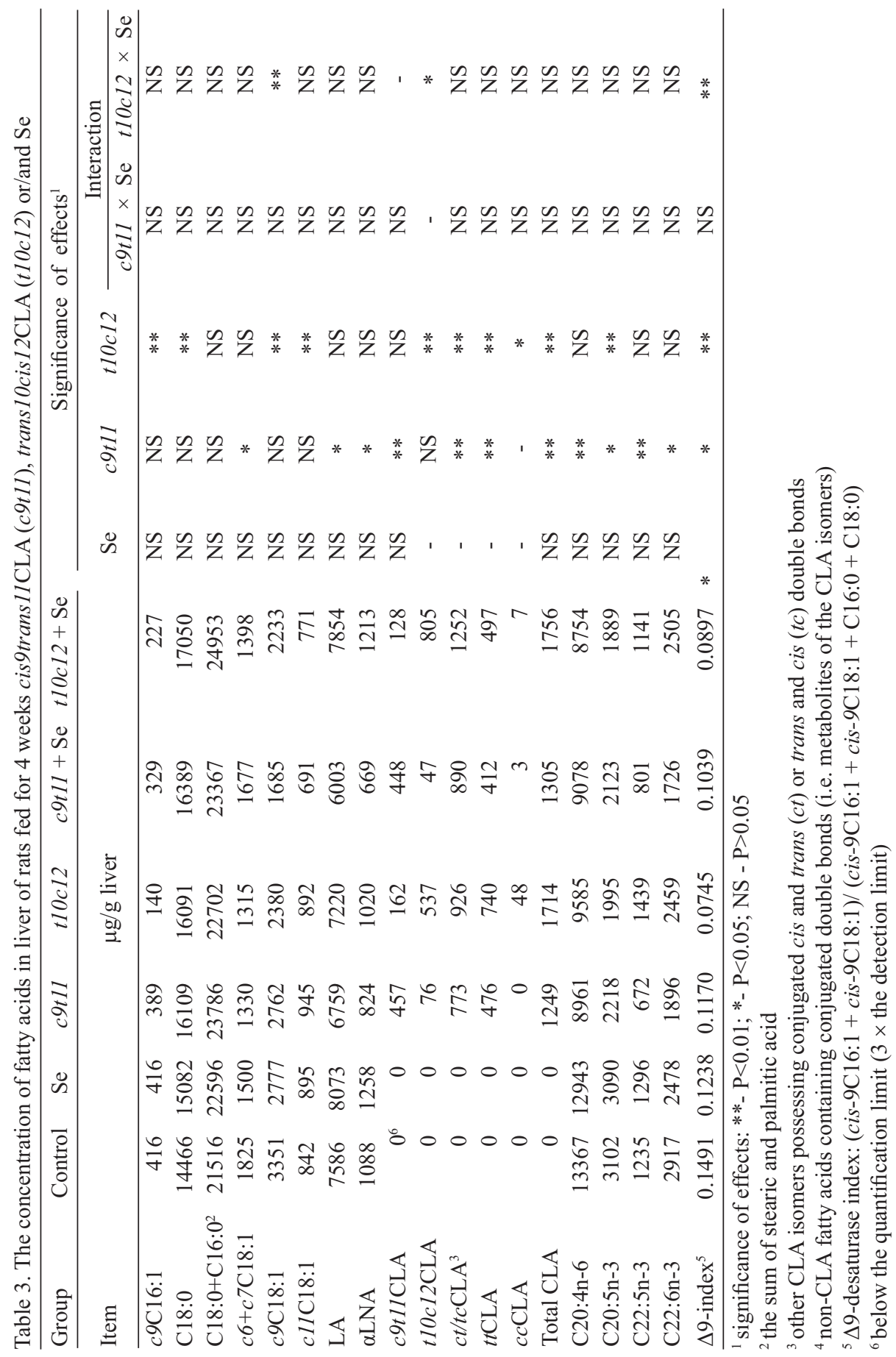




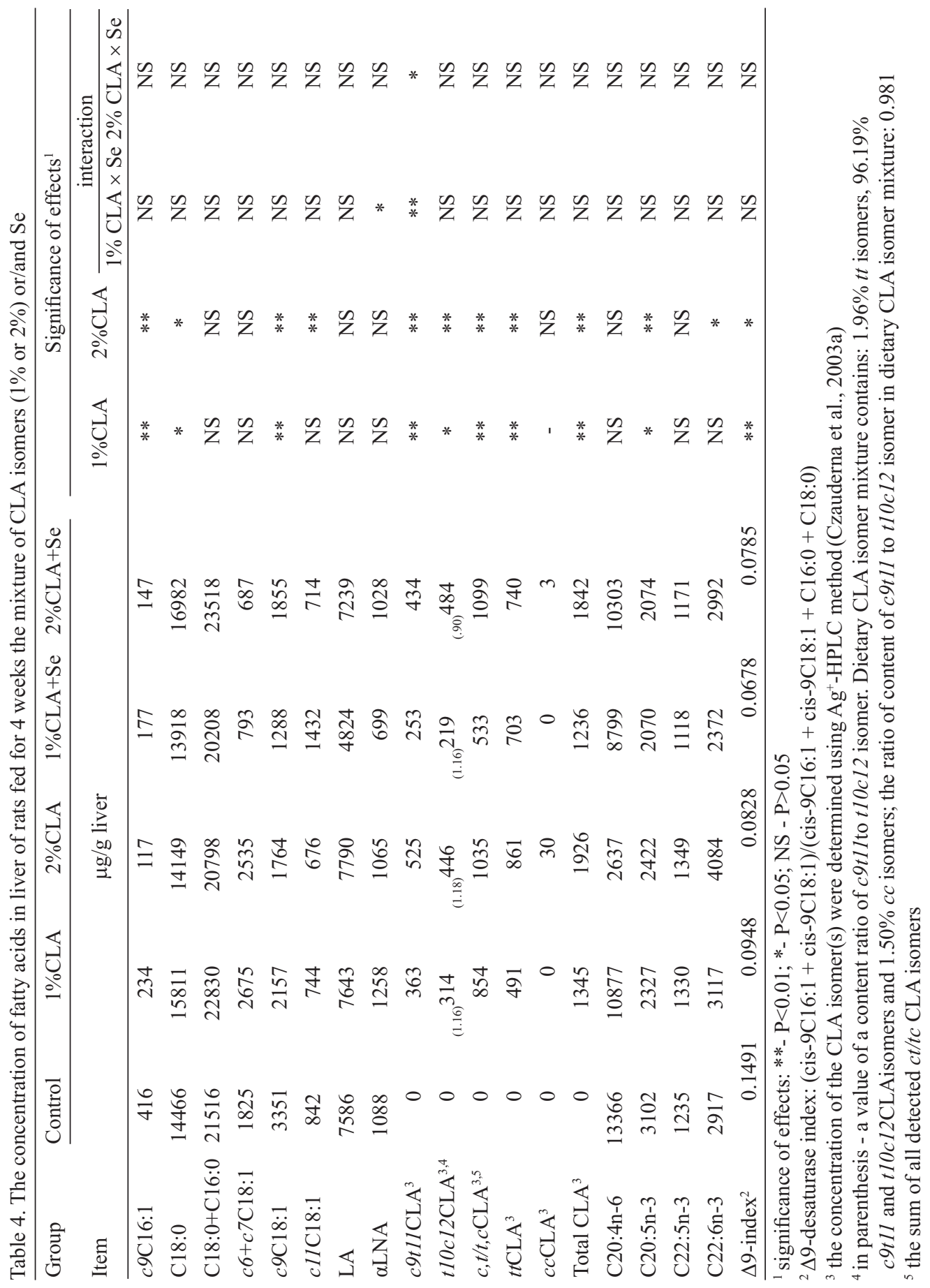


ratio of these isomers in the liver of rats fed the CLA isomer mixtures $(\mathrm{R}=1.16$ - 1.18; Table 4). The presence of Se in the diets enriched to a higher level of CLA isomers $(2 \%)$ considerably increased the accumulation of $t 10 \mathrm{c} 12 \mathrm{CLA}$ in the liver. Consequently, the value of $\mathrm{R}_{c 9 t 1 / \mathrm{CLA} / t 10 c \mid 2 \mathrm{CLA}}$ in the liver of rats fed the $2 \%$ CLA isomer mixture and Se was significantly lower compared with the value of the ratio of these isomers in the dosed CLA isomer mixture and in the liver of rats fed the diets with only CLA isomer(s). The present results are in agreement with our previous studies in which dietary selenate also decreased the yield of preferential $\beta$-oxidation of $110 c 12 \mathrm{CLA}$ (Czauderna et al., 2004a,b), however, dietary organic Se more efficiently inhibited the capacity of $\beta$-oxidation of this isomer (Tables 3 and 4) compared with inorganic Se.

The current study clearly indicates that trans, transCLA isomers are preferentially incorporated into the liver, whereas there is partial discrimination in the accumulation of cis, cis and cis,trans/trans, cisCLA isomers. The lower accumulation of cis,trans/ trans,cis and cis,cis is the result of rapid metabolism, $\beta$-oxidation (particularly of the $110 \mathrm{c} 11 \mathrm{CLA}$ isomer) and unfavourable geometrical configuration. In contrast, more efficient accumulation of trans, transCLA isomers could be due to their slower metabolism and $\beta$-oxidation as well as preferred geometrical insertion into membrane phospholipids.

As expected, feeding the CLA isomer mixtures (1 or $2 \%$ ) or individual CLA isomers, particularly $t 10 c 12 \mathrm{CLA}$, irrespective of the presence of Se, resulted in decreasing the content of oleic acid (cis-9C18:1) and cis-9C16:1 in the liver. This is consistent with our previous results (Czauderna et al., 2003b, 2004a, b) showing that the CLA isomers, especially 110 c12CLA, are responsible for the change in the levels of monounsaturated and saturated fatty acids in organs from animals fed CLA isomers due to decreasing the $\Delta$ 9-desaturation of FAs such as C16:0 or C18:0 (Alasnier et al., 2002). Recent studies (Alasnier et al., 2002; Kang et al., 2004) have demonstrated that CLA isomers, particularly $t 10 c 12$ CLA, caused a reduction in $\Delta 9$ desaturase capacity, inhibited stearoyl-CoA desaturase mRNA expression and fatty acid synthesis (Belury, 2002). Consequently, the value of the $\Delta 9$-desaturase index in the liver of rats fed a diet enriched in CLA isomers decreased compared with that in the liver of control rats (Tables 3 and 4). The experimental diet enriched only in Se also caused a reduction in $\Delta 9$-desaturase capacity, inhibited stearoyl-CoA desaturase mRNA expression (Table 3).

The addition of organic Se (mainly as Se-Met) to the diet resulted in decreasing the concentration of $c i s-9 \mathrm{C} 18: 1$ in rat liver. Our current results are in agreement with our previous studies in which inorganic Se (as selenate) also efficiently decreased the concentration of cis-9C18:1 in the liver, femoral muscles and perigonadal fat of experimental rats (Czauderna et al., 2003b, 2004a,b). Se supplementation to the diet enriched in the CLA isomer mixture (1 and 2\%) or c9t11CLA resulted in a 
numerical decrease in the concentrations of linoleic (C18:2n-6; LA) and linolenic (C18:3n-3; $\alpha$ LNA) acids in the liver compared with rats fed with the CLA isomer mixtures or $c 9 t 11$ CLA (Tables 3 and 4). On the other hand, $c 9 t 11$ CLA in the diet most efficiently decreased the concentration of LA and $\alpha$ LNA in the liver compared with the control group or rats fed the diet enriched in only t10c12CLA (Table 3). These results indicate that the individual CLA isomers, particularly c9t11CLA, statistically or numerically decreased $\Delta 6$-desaturase capacity, while, surprisingly, the mutual interaction between dosed $t 10 c 12 \mathrm{CLA}$ and $c 9 t 11 \mathrm{CLA}$ tended to slightly increase the capacity of this desaturase. Thus, our current study is in agreement with our previous investigation (Czauderna et al., 2004b) in which the individual CLA isomers decreased $\Delta 6$-desaturase capacity, whereas the mixture of the CLA isomers increased the capacity of this desaturase in the liver of rats.

Detailed investigations of the concentration of stearic and palmitic acids in the rat liver reinforce the findings of this study and of Banni et al. (2004) showing that the $c 9 t 11$ isomer and $t 10 c 10 \mathrm{CLA}$, in particular, decrease the yield of cis-9 fatty acids formation (Table 3). Consequently, the concentrations of these saturated fatty acids are higher in the liver of rats fed the diet enriched in these isomers, irrespective of the presence of Se, compared with the controls. However, feeding the CLA isomer mixture (1 or 2\%) with or without the presence of Se resulted in an inconsistent effect of the administered CLA isomer mixture on the concentration of stearic and palmitic acids in the liver. We hypothesize that this irregular effect of these additives to the rat diet is due to interactions between CLA isomers as well as dosed Se (Table 4).

In the present study, feeding the CLA isomer mixtures (1 or 2\%) with or without Se affected the concentration of C20:4n-6, C20:5n-3, C22:5n-3 and C22:6n-3 in the rat liver (Table 4), whereas administration of individual CLA isomers, regardless of the addition of Se, usually numerically or significantly decreased the concentration of these polyunsaturated fatty acids in the rat liver (Table 3). Surprisingly, feeding the CLA isomer mixture (1 and 2\%) with or without $\mathrm{Se}$ decreased numerically or significantly the concentration of only C20:4n-6 and C20:5n-3 in the liver of experimental rats. The diet enriched in the CLA isomer mixture (1 and 2\%) increased the concentration of C22:5n-3 and C22:6n-3 in the liver and this effect was stronger as the concentration of the CLA isomer mixture in the diet increased (i.e. to $2 \%$ ). Addition of Se to the diet enriched in the CLA isomer mixture reduced the observed influence of an isomer mixture on the concentration of these fatty acids; consequently the concentration of C22:5n-3 and C22:6n-3 in the liver was slightly lower compared with the concentrations in the liver of the control group or rats fed only the CLA isomer mixtures (Table 4). This influence of added Se is weaker as the concentration of the CLA isomer mixture in the diet is higher $(2 \%)$. Therefore, we suggest that the diet enriched in the mixture of the 
CLA isomers increased the yield of the elongation of C20:5n-3 and the capacity of $\Delta 4$-desaturase in the liver in comparison with the control group. In contrast, the CLA isomer mixture, irrespective of the presence of Se, caused a decrease in the concentration of C20:4n-6 and C20:5n-3 in the liver, therefore we conclude that administering the CLA isomer mixture to the diet reduced the capacity of $\Delta 5$ desaturase. In addition, supplementing the individual CLA isomers, regardless of the presence of Se, usually numerically or significantly decreased the yield of the elongation of $\mathrm{C} 18: 4 n-3$, and $\mathrm{C} 18: 3 n-6$, and the capacity of $\Delta 6-$ and $\Delta 5$ desaturases in the liver. On the other hand, the diet enriched in only Se had a negligible influence on the concentration of C20:4n-6, C20:5n-3, C22:5n-3 and $\mathrm{C} 22: 6 \mathrm{n}-3$ in the liver compared with the control group.

Table 5. The concentration of triacylglycerols (TAG), total (TC), HDL ${ }^{1}$ fraction of cholesterol and $\mathrm{LDL}^{2}$ fraction of cholesterol in blood plasma of rats feed CLA isomer(s) or Se

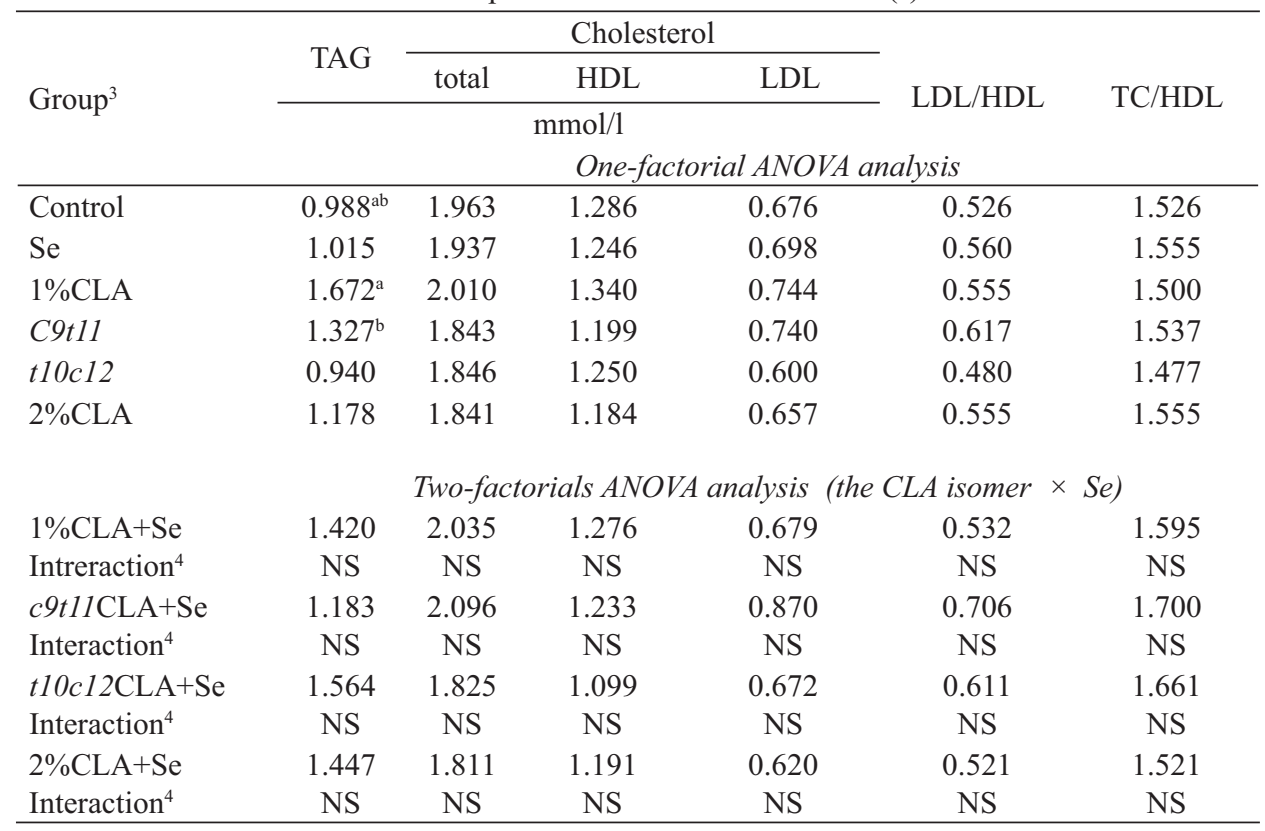

${ }^{1}$ high-density lipoprotein

2 light-density lipoprotein

${ }^{3}$ means in columns with the same letter are significantly different: ${ }^{\mathrm{a}, \mathrm{b}}-\mathrm{P}<0.05$

${ }^{4}$ interaction: data were analyzed by two-factorials: the CLA isomer(s) $\times \mathrm{Se}$; statistical significance was declared at $*$ - $\mathrm{P}<0.05$ and $* *-\mathrm{P}<0.01 ; \mathrm{NS}$ - not significant (ANOVA test)

The results of the dose-response changes in triacylglycerols (TAG) and cholesterol fraction concentrations in blood plasma by different forms of additives are summarized in Table 5. It was found that there was a numerical decrease the 
concentration of total cholesterol (TC) and the HDL cholesterol fraction in plasma of rats fed the diet enriched in Se only. Indeed, there is recent evidence that excess Se supplementation ( $5 \mu \mathrm{g} / \mathrm{kg}$ diet) significantly decreases TC in rabbits (Vinson et al., 1998). In our study, dietary c9t11CLA and $t 10 c 12$ CLA numerically decreased the concentration of TC and HDL cholesterol, whereas supplementing t10c11CLA resulted in the most efficient decrease in the LDL concentration. The diet enriched in the higher concentration of the CLA isomer mixture most efficiently decreased the concentration of TC and HDL cholesterol in blood plasma of rats. The mixture of CLA isomers at a dose of $1 \%$ numerically or significantly increased the concentration of HDL and LDL cholesterol as well as the content of TCG and TC. On the other hand, administering $t 10 c 12 \mathrm{CLA}$ as well as the mixture of the CLA isomers at a dose of $2 \%$ decreased the concentration of the HDL and LDL fractions in blood plasma. Surprisingly, in contrast to the recent results of Tricon et al. (2004), in our current study administering $110 c 12$ CLA revealed a tendency towards lowering the LDL:HDL concentration ratio as well as the value of the TC: HDL ratio as compared with the control group and all other experimental diets. Furthermore, in our study, the diet with $t 10 c 12 \mathrm{CLA}$ most efficiently numerically decreased the concentration of TCG in plasma compared with the control group. The possible explanation is that $110 \mathrm{c} 12 \mathrm{CLA}$ treatment stimulated fatty acid oxidation, reduced body fat accretion with concurrent increases in lean tissue deposition (Belury, 2002). This is valuable information for nutritionists in the context carrying out research to improve the nutritional quality of food for human health.

\section{CONCLUSIONS}

The current study revealed that in rats, during feeding diets enriched in CLA isomer(s) with or without Se for 4 weeks, a major increase in the concentration of CLA isomer(s) occurred in the liver. Dietary 110 c12CLA and Se significantly enhanced the body weight accretion of rats, the concentration of CLA isomer(s) in the liver and muscles, as well as feed conversion efficiency. Adding $1.2 \mathrm{ppm} \mathrm{Se}$ with or without the CLA isomer(s) increased the content of the essential element (Se) in the body of experimental animals (without toxicity), thus, supplementing Se with or without the CLA isomer(s) improves the nutritional quality of products derived from animals to a greater degree. The supplemented CLA isomer mixture elevated the liver concentration of n-3 long-chain polyunsaturated fatty acids (i.e. C22:5n-3 and C22:6n-3) and this effect was stronger as the level of CLA in the diet increased. Addition of Se to the diets containing the CLA isomer(s) at the dose of $1 \%$ documented a tendency toward increasing the values of the plasma LDL: 
HDL and TC:HDL ratios. Moreover, the diet enriched in only Se also resulted in increases in the values of these ratios and the concentration of LDL cholesterol and TAG.

These results constitute valuable information for nutritionists in the context of carrying out research to improve the nutritional quality of food for domestic animals and human health.

\section{ACKNOWLEDGEMENTS}

The authors are very grateful to Łukasz Jedynak for calculating the concentrations of $\mathrm{Zn}, \mathrm{Fe}, \mathrm{Mg}, \mathrm{Ca}$ and $\mathrm{Cu}$ in liver and muscles.

\section{REFERENCES}

Akahoshi A., Koba K., Ohkura-Kaku S., Kaneda N., Goto C., Sano H., Iwata T., Yamauchi Y., Tsutsumi K., Sugano M., 2003. Metabolic effects of dietary conjugated linoleic acid (CLA) in rats. Nutr. Res. 23, 1691-1701

Alasnier C., Berdeaux O., Chardigny J.M., Sébédio J.L., 2002. Fatty acid composition and conjugated linoleic acid content of different tissues in rats fed individual conjugated linoleic acid isomers given as triacylglycerols. J. Nutr. Biochem. 13, 337-345

Banni S., Petronib A., Blasevichb M., Cartaa G., Angionia E., Murrua E., Dayc B.W., Melisa M.P., Spadaa S., Clement C., 2004. Detection of conjugated C16 PUFAs in rat tissues as possible partial beta-oxidation products of naturally occurring conjugated linoleic acid and its metabolites. Biochim. Biophys. Acta 1682, 120-127

Belury M.A., 2002. Dietary conjugated linoleic acids in health: Physiological effects and mechanisms of action. Annu. Rev. Nutr. 22, 505-531

Choi Y., Kim Y.-C., Han Y.-B., Park Y., Pariza M.W., Ntambi J.M., 2000. The trans-10, cis-12 isomer of conjugated linoleic acid downregulates stearoyl-CoA desaturase 1 gene expression in 3T3-L1 adipocytes. J. Nutr. 130, 1920-1924

Czauderna M., Kowalczyk J., Korniluk K., Wąsowska I., 2005. Improving the analysis of fatty acids using combination of gas chromatography and $\mathrm{Ag}^{+}$liquid chromatography. J. Anim. Feed Sci. 14, Suppl. 1, 263-266

Czauderna M., Kowalczyk J., Niedźwiedzka K.M., Wąsowska I., Pastuszewska B., 2004a. Conjugated linoleic acid (CLA) content and fatty acids composition of muscle in rats fed isomers of CLA and selenium. J. Anim. Feed. Sci. 13, 183-196

Czauderna M., Kowalczyk J., Niedźwiedzka K.M., Wąsowska I., Pastuszewska B., Bulska E., Ruszczyńska A., 2004b. Liver and body mass gain, content of CLA isomers and other fatty acids in the liver of rats fed CLA isomers and selenium. J. Anim. Feed. Sci. 13, 353-369

Czauderna M., Kowalczyk J., Wąsowska I., Niedźwiedzka K.M., 2003a. Determination of conjugated linoleic acid isomers by liquid chromatography and photodiode array detection. J. Anim. Feed Sci. 12, 269-282

Czauderna M., Kowalczyk J., Wąsowska I., Niedźwiedzka K.M., Pastuszewska B., 2003b. The effects of selenium and conjugated linoleic acid (CLA) isomers on fatty acid composition, CLA isomer content in tissues, and growth of rats. J. Anim. Feed Sci. 12, 865-881 
Degrace P., Demizieux L., Gresti J., Chardigny J., Sébédio J., Clouet P., 2003. Association of liver steatosis with lipid oversecretion and hypotriglyceridaemia in C57BL/6j mice fed trans-10, cis12-linoleic acid. FEBS Lett. 546, 335-339

Gnädig S., Rickert R., Sébédio J.L., Steinhart H., 2001. Conjugated linoleic acid (CLA): physiological effects and production. Eur. J. Lipid Sci. Tech. 103, 56-61

Kang K., Miyazaki M., James M., Ntambi J.M., Pariza M.W., 2004. Evidence that the anti-obesity effect of conjugated linoleic acid is independent of effects on stearoyl-CoA desaturase1 expression and enzyme activity. Biochem. Biophys. Res. Commun. 315, 532-537

Park Y., Storksonb J.M., Albrightb K.J., Liub W., Pariza M.W., 2005. Biological activities of

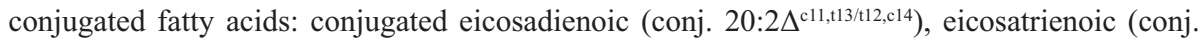

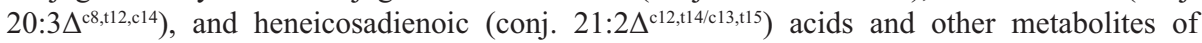
conjugated linoleic acid. Biochim. Biophys. Acta 1687, 120-129

Rainer L., Heiss C., 2004. Conjugated linoleic acid: health implications and effects on body composition. J. Amer. Diet. Assn. 104, 963-968

Rayman P.M., 2004. Review article. The use of high-selenium yeast to raise selenium status: how does it measure up? Brit. J. Nutr. 92, 557-573

Simon O., Männer K., Schäfer K., Sagredos A., Eder K., 2000. Effects of conjugated linoleic acids on protein to fat proportions, fatty acids, and plasma lipids in broilers. Eur. J. Lipid Sci. Tech. $102,402-410$

Suzuki K.T., 2005. Metabolomics of selenium: Se metabolites based on speciation studies. J. Health Sci. 51, 107-114

Tapiero H., Townsend D.M., Tew K.D., 2003. The antioxidant role of selenium and selenocompounds. Biomed. Pharmacother. 57, 134-144

Terpstra A.H.M., 2003. Fat and Energy Metabolism. An Overview of the Literature. Department of Laboratory Animal Science, Utrecht University Press, Utrecht (The Netherlands)

Tricon S., Burge G.C., Kew S., Banerjee T., Russell J.J., Jones E.L., Grimble R.F., Williams C.M., Yaqoob P., Calder P.C., 2004. Opposing effect of cis-9,trans-11 and trans-10,cis-12 conjugated linoleic acid on blood lipids in healthy humans. Amer. J. Clin. Nutr. 80, 614-620

Vinson J.A., Stella J.M., Flanagan T.J., 1998. Selenium yeast is an effective in vitro anti-oxidant and hypolipemic agent in normal hamster. Nutr. Res. 18, 735-742

Wahle K.W.J., Steven D., Heys S.D., Rotondo D., 2004. Conjugated linoleic acids: are they beneficial or detrimental to health? Prog. Lipid Res. 43, 553-587

Whanger P.D., 2004. Selenium and its relationship to cancer: an update. Brit. J. Nutr. 91, 11-28 